\title{
Effect of photoperiod on the annual cycle of testis growth in a tropical mammal, the little red flying fox, Pteropus scapulatus
}

\author{
G. M. O'Brien, J. D. Curlewis and L. Martin \\ Department of Physiology and Pharmacology, The University of Queensland, Queensland 4072, \\ Australia
}

\begin{abstract}
Little red flying foxes (Pteropus scapulatus) are seasonal breeders: they mate in late spring/ early summer, and young are born the following autumn. In captivity, males housed outdoors in a normal breeding colony in natural daylight showed a single cycle of testis growth and regression each year. During reproductive quiescence, testicular volume was approximately $2 \mathrm{~cm}^{3}$; recrudescence began soon after the winter solstice; testicular volume was maximum at approximately $6.5 \mathrm{~cm}^{3}$ at the spring equinox; and regression was complete by the end of summer. To test whether photoperiod entrains or synchronizes the cycle, groups of males were housed indoors, without females, at constant temperature, and artificial lighting was timed to either mimic naturally changing daylength or provide alternating 3-month periods of short ( $11 \mathrm{~h}$ light: $13 \mathrm{~h}$ dark) or long ( $16 \mathrm{~h}$ light: $8 \mathrm{~h}$ dark) days (two groups, three months out of phase with each other). During 18 months, the applied photoperiod protocol had no effect on the frequency of testicular cycles (which remained at one per year), the time course of recrudescence and regression (as described above for normal outdoor control males), or the completeness of growth and regression stages. These results suggest that male $P$. scapulatus are not reproductively photoresponsive.
\end{abstract}

\section{Introduction}

Pteropus scapulatus, little red flying foxes (Family Pteropodidae, suborder Megachiroptera), are seasonal breeders. In captivity, individuals copulate repeatedly during several months, and peak mating occurs in November and December (spring/ summer) (G. M. O'Brien and R. F. Nankervis, unpublished), with births occurring about 6 months later (G. M. O'Brien, R. F. Nankervis and L. Martin, unpublished). The few reports of wild populations indicate that there are no time differences between reproduction in captive and free living populations (G. M. O'Brien et al., unpublished). P. scapulatus are nomadic, following food resources (Ratcliffe, 1931; Nelson, 1965; McWilliam, 1985). In winter they are widely distributed in tropical northern Australia (approximately $10-25^{\circ} \mathrm{S}$ ), whereas from late spring to early autumn their distribution extends through coastal regions, to the southern part of mainland Australia $\left(35^{\circ} \mathrm{S}\right)$. Although often found in coastal habitats they also follow watercourses deep inland at times (Hall, 1987). Preliminary studies have shown that there is a seasonal change in testis activity in the male, with a peak in spring (McGuckin and Blackshaw, 1991); however, the environmental factors that synchronize this cycle are unknown.

P. poliocephalus, the grey-headed flying fox, inhabits forests and mangroves of south-eastern Australia (20-40 ${ }^{\circ} \mathrm{S}$ : Hall, 1987) and McGuckin and Blackshaw (1992) found that reproduction in male P. poliocephalus could be influenced by photoperiod. Compared with $P$. poliocephalus, $P$. scapulatus has a small pineal gland (McGuckin and Blackshaw, 1988; Bhatnagar et al., 1990).

Received 29 May 1992.
This led McGuckin (1988) to suggest that reproduction in $P$. scapulatus might be regulated by temperature rather than photoperiod. To investigate further the regulation of seasonal reproduction in $P$. scapulatus, we tested whether altered photoperiod could disrupt the cycle of gonadal growth and regression.

\section{Methods}

\section{Animals and husbandry}

Mature adult male $P$. scapulatus (Fig. 1) (ages unknown) were collected by hand from camps in mangroves or low eucalypt forest in Queensland, Australia, and housed at the flying fox research colony of the University of Queensland, Brisbane (latitude $28^{\circ} \mathrm{S}$ ). Food was provided fresh daily ad libitum (approximately $300 \mathrm{~g}$ each) as a mixture of diced $(1.5 \mathrm{~cm})$ fruits predominantly apple, pear, pawpaw (papaya), melons, mango, banana, avocado, grapes and stonefruit. Since the animals did not have access to their native diet, predominantly blossom (Ratcliffe, 1931; Nelson, 1965), they were offered a milk supplement (Denkavit Calf Milk, Murray Goulburn Co-operative Co. Ltd, Melbourne) and a vitamin and mineral enriched molasses block (Rumevite Horse and Goat Block, Cheetham Rural, Wacol, Queensland) as well as fresh water.

\section{Testis cycle under natural photoperiod}

The first experiment aimed to determine the annual cycle of testicular growth in $P$. scapulatus, particularly its time course, 


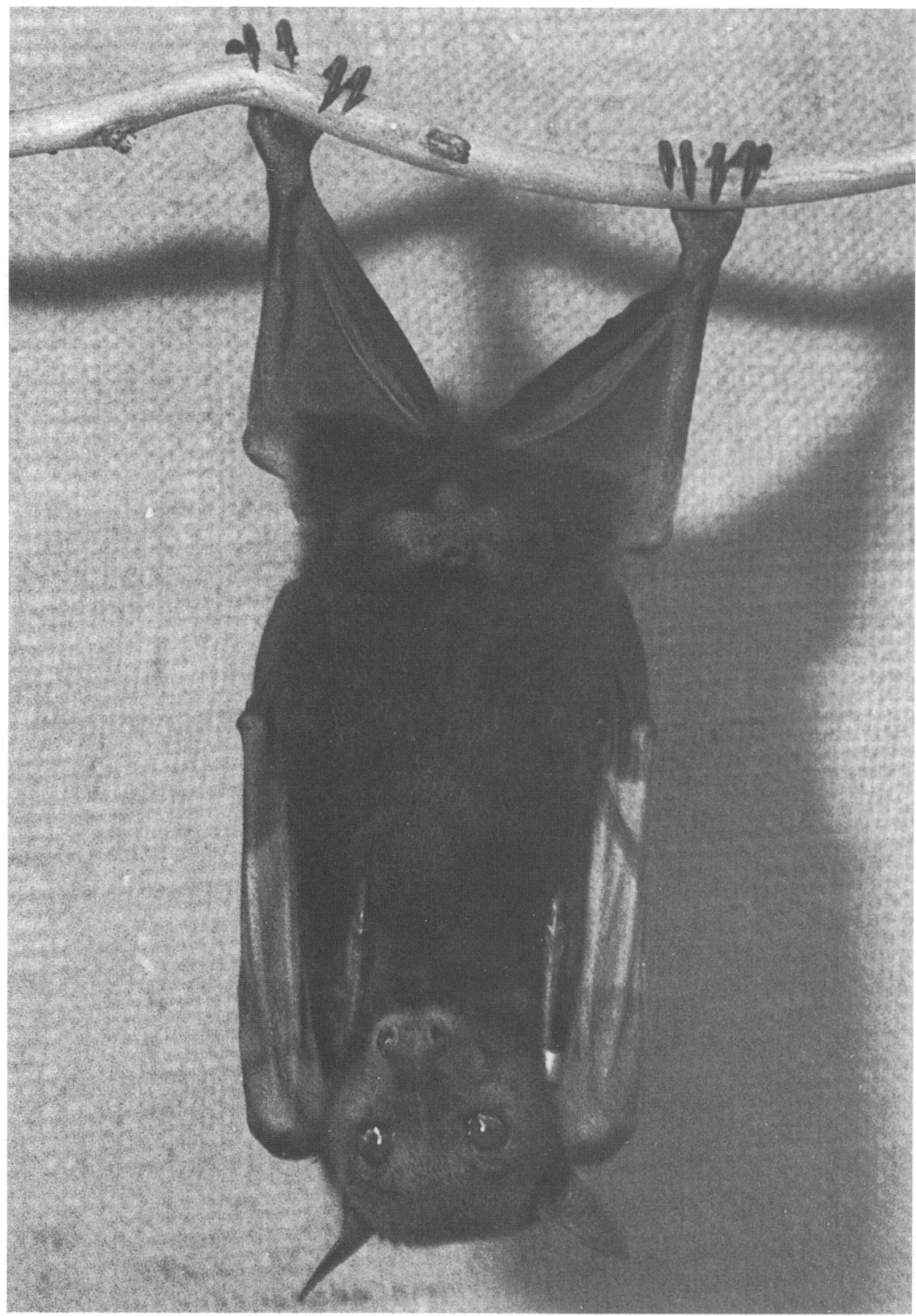

Fig. 1. Adult, male, little red flying fox, Pteropus scapulatus, photographed in August in the non-breeding period. The scrotal testes are well presented for measurement.

amplitude and the biological variability between individuals and between years. For this, animals were monitored for 2.25 years, while housed in a breeding group, in naturally changing daylength and temperature.

These animals were part of the normal breeding colony, comprising up to 8 males and 19 females, and had been in captivity for some time (several months to years) before this study began. They were housed in the open, in a galvanized wire mesh enclosure $(2 \mathrm{~m} \times 2 \mathrm{~m} \times 6 \mathrm{~m})$ with several feeding stations, and an insulated shelter area at one end with curtains for privacy and protection from weather, mainly wind. Maximum and minimum temperatures, recorded by the Bureau of Meteorology at the nearest appropriate weather station, are presented (Fig. 2).
However, in winter, the animals had access to a section of the shelter area that was warmed at night (with a column oil heater) to prevent the minimum temperature falling too low.

\section{Effect of photoperiod manipulation}

The second experiment aimed to determine whether altered photoperiods could disrupt the annual cycle of testicular growth. Males were housed indoors, without females, in artificial lighting and regulated temperature (approximately $20^{\circ} \mathrm{C}$ ). Group A (indoor controls) were kept under a simulated natural photoperiod, although technical difficulties altered the symmetry during the second 6 months. Times of lights-on and 

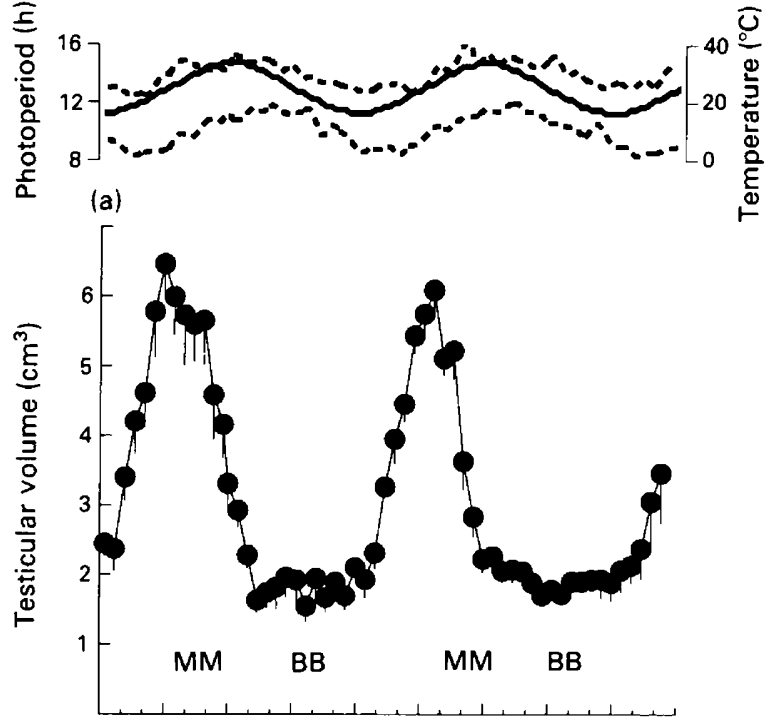

(b)

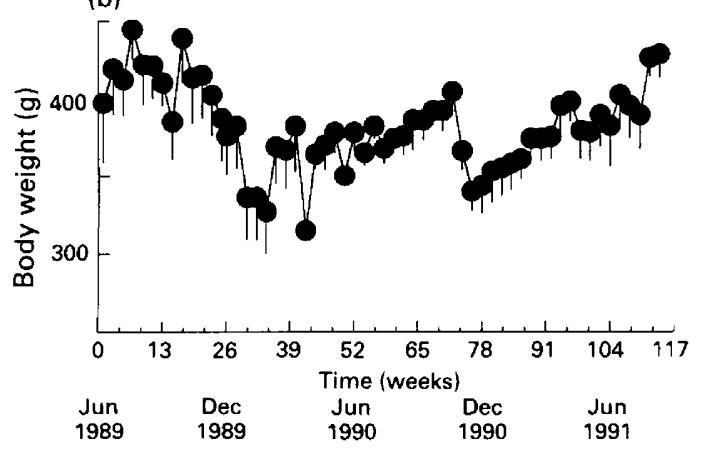

Fig. 2. Mean ( \pm SEM) (a) testis volume and (b) body weight of normal breeding $P$. scapulatus monitored every two weeks for 2.25 years, from June 1989. ( $\longrightarrow$ ) Photoperiod; (-- ) maximum and minimum temperatures; MM: mating period (November-December); BB: births in captivity (April-May).

lights-off were calculated from the times of sunrise and sunset, with an additional 30 min added at each end of the photophase to allow for twilight: this gave a daylength range of $11 \mathrm{~h} 15 \mathrm{~min}$ to $14 \mathrm{~h} 45 \mathrm{~min}$, and a total cycle duration of 12 months. Groups $B$ and $C$ were exposed to alternating 3 month periods of short (11 $\mathrm{h}$ light: $13 \mathrm{~h}$ dark) and long ( $16 \mathrm{~h}$ light: $8 \mathrm{~h}$ dark) daylengths, a total cycle length of 6 months. The applied photoperiod therefore had a frequency of two cycles per year. Group B began with short days and Group $C$ with long days. Thus, the photoperiods for groups $\mathrm{B}$ and $\mathrm{C}$ were out of phase. During the first 6 months, short days were $8 \mathrm{~h}$ light:16 h dark for adaptation. The protocol was maintained for 18 months from the winter solstice in June 1989 until the summer solstice in December 1990.

If animals housed under opposite photoperiods showed testis cycles that were out of phase (with each other) then photoperiodic control of gonadal growth is implicated; if, however, testis changes remained synchronous between groups then a factor other than photoperiod (e.g. endogenous rhythm) would be implicated. Group A differed from Groups B and C in photoperiod only, providing control data for possible effects of isolating small groups of males, without females, in artificial lighting and regulated temperature.

Animals in the photoperiod manipulation experiment were collected from a single, flying fox camp approximately $300 \mathrm{~km}$ north of Brisbane in June 1989. After one week in captivity, they were allocated to groups and placed into $2 \mathrm{~m} \times 2 \mathrm{~m} \times$ $1.5 \mathrm{~m}$ wire mesh enclosures that had been built inside closed, insulated rooms. Each room was independently ventilated by reverse cycle air conditioners, each with separate air inlets from outside to reduce transfer of chemical information between groups; and each had a maximum-minimum thermometer that was read and reset every two weeks. Illumination was from two ceiling mounted 36 watt triphosphor tubes (daylight wavelengths, $6500 \mathrm{~K}$; Philips Australia) switched via timers that could be adjusted by $15 \mathrm{~min}$ increments. An incandescent globe ( $40 \mathrm{~W}$ red) in each room and service passage permitted entry at any time without interfering with light cycles, as well as providing low light for these nocturnal animals. The diurnal variation in light intensity, measured at head height of the flying foxes, was $1800 \mathrm{~lx}$ (lights on) to $2-15 \mathrm{~lx}$ (lights off i.e. red lights only).

\section{Collection of data}

Testes (in the scrotum) were measured using vernier or digital callipers: testis volume was calculated from the length and width of each testis using the formula for a prolate sphere; data reported below are the combined testicular volume for paired testes. Body weight and testicular volume were recorded every two weeks. Time, in weeks, was counted from the winter solstice, 1989.

While $P$. poliocephalus adapt well to captivity and handling, it is our experience that $P$. scapulatus continue to have relatively poor rates of reproduction and long-term survival in captivity (normal lifespan has not yet been determined). Although the normal outdoor breeding colony is increasingly successful, it had to be assumed that moving animals indoors posed an added risk to the condition of the animals. For this reason, the protocol was designed with a minimum of invasive procedures: no blood samples were collected, and therefore plasma testosterone concentrations were not measured.

\section{Groups}

The experiments were established with 6-8 males per group. In the first year, one animal died of acute illness (septicaemia) and four died from general adaptation syndrome (Selye, 1953), apparently associated with living in captivity, and possibly confounded by age. Their data have been excluded. For most of the experiment, numbers remained at five in the outdoor, breeding group, and seven, six and four in Groups A, B and C, respectively. When the alpha male (judged from his dominant and assertive behaviour, and size) in the breeding group was taken by a carpet snake (Python spilotus variegatus), the animal that appeared to replace him as alpha male was inducted into the study group to avoid bias. In the final 6 months there were a further five acute deaths (general adaptation syndrome (three), accident (one) and one of unknown cause) and one animal was removed for adaptation to other conditions. Thus at the end of the experiment, there were five animals in the breeding group, 
Table 1. Summary of testicular volume (TV) at peak and nadir of reproductive cycles of Pteropus scapulatus, and times at which they occurred

\begin{tabular}{|c|c|c|c|c|}
\hline & \multirow{2}{*}{$\begin{array}{l}\text { Breeding } \\
\text { group }\end{array}$} & \multicolumn{3}{|c|}{ Photoperiod manipulation } \\
\hline & & Group A & Group B & Group C \\
\hline \multicolumn{5}{|l|}{ First year (1989) } \\
\hline Baseline TV $\left(\mathrm{cm}^{3}\right)$ & $2.2 \pm 0.2$ & $1.7 \pm 0.1^{a}$ & $1.9 \pm 0.1^{\mathrm{a}}$ & $1.7 \pm 0.2^{\mathrm{a}}$ \\
\hline Maximum TV $\left(\mathrm{cm}^{3}\right)$ & $7.0 \pm 0.6$ & $5.1 \pm 0.4^{a}$ & $4.0 \pm 0.4^{\mathrm{a}}$ & $4.8 \pm 0.2^{\mathrm{a}}$ \\
\hline Week maximum TV achieved & $15 \pm 1$ & $12 \pm 1^{\mathrm{a}}$ & $12 \pm 1^{a}$ & $12 \pm 1^{a}$ \\
\hline Baseline TV (weeks 33-44) & $1.8 \pm 0.2$ & $2.4 \pm 0.1^{\mathrm{a}}$ & $2.6 \pm 0.3^{\mathrm{a}}$ & $2.9 \pm 0.5^{\mathrm{a}}$ \\
\hline$n$ & 6 & 7 & 6 & 4 \\
\hline \multicolumn{5}{|l|}{ Second Year (1990) } \\
\hline Maximum TV $\left(\mathrm{cm}^{3}\right)$ & $6.1 \pm 0.4$ & $3.8 \pm 0.3^{\mathrm{a}}$ & $4.4 \pm 0.3^{\mathrm{a}}$ & $5.9 \pm 0.1^{b}$ \\
\hline Week maximum TV achieved & $67 \pm 1$ & $66 \pm 1^{3}$ & $66 \pm 1^{a}$ & $65 \pm 3^{a}$ \\
\hline Baseline TV (weeks 85-96) & $1.8 \pm 0.1$ & - & - & - \\
\hline$n$ & 5 & 6 & 6 & 3 \\
\hline
\end{tabular}

Results are means \pm SEM based on data from individuals.

a,b Means with the same letter are not significantly different, between groups (within year) at $P<0.01$, Duncan's Multiple Range test.

Group A: indoor, natural photoperiod; Groups B and C exposed to 3 month periods of short ( $11 \mathrm{~h}$ light: $13 \mathrm{~h}$ dark) and long ( $16 \mathrm{~h}$ light:8 $\mathrm{h}$ dark) daylengths (Group B began with short days and Group $\mathrm{C}$ began with long days).

and three, five and two in Groups A, B and C, respectively. At the completion of the experiment all animals were returned to normal outdoor enclosures.

\section{Statistical analysis}

Data were analysed by analysis of variance using SAS/STAT General Linear Modelling Procedure (SAS, 1985). Data that were significantly different were ranked using Duncan's Multiple Range Test.

\section{Results}

\section{Testis cycle under natural photoperiod}

Adult male $P$. scapulatus housed with females in natural light and temperature initiated testicular recrudescence 3-4 weeks after the winter solstice (Fig. 2). Maximum testis volume was achieved by week 15 (Table 1), in October, and the subsequent regression was completed by a few weeks after the summer solstice (Fig. 2a). The period of the cycle was 52 weeks, peak to peak. There was no difference between years with respect to the maximum testis volume $(P>0.6)$, baseline testis volume $(P>0.6)$, or the week in which maximum testicular volume was achieved $(P>0.6)$.

Body weight, monitored every two weeks, displayed an apparently regular pattern in both years (Fig. 2b). In the first year animals shed weight in association with breeding, and increased body weight the following year after testicular regression was complete. They again lost weight at the time of the breeding season in 1990 .

\section{Effect of manipulation of photoperiod}

All groups of animals in artificial photoperiod and temperature underwent essentially similar cycles of testicular growth and regression (Fig. 3). The period of the cycle was approximately one year (54, 54 and 53 weeks, peak to peak, in Groups A-C) irrespective of treatment (Fig. 3 and Table 1). There were no differences between groups with respect to the number of testicular cycles per year or the time course of events in the testicular cycle under any treatment (Table 1 ).

Body weights were very variable, with some apparent pattern differences between groups (Fig. 4). Group A maintained body weight at the time of the (1989, outdoor) breeding season, then expressed the following autumn increase and the subsequent weight loss at the time of the (1990, outdoor) breeding season. Groups B and C lost weight when first moved into captivity and artificial housing, but after the (outdoor) breeding season their body weights recovered, and in the second year showed signs of expressing a pattern similar to that of the outdoor, breeding group (and Group $A$ in the second year).

\section{Discussion}

Male $P$. scapulatus displayed a single, large amplitude cycle of testis growth and regression each year. Recrudescence began shortly after the winter solstice; maximum testicular volume was achieved at about the time of the spring equinox; and regression was complete by the end of summer. Minimum testis size was maintained from when regression was complete at 2 to 6 weeks after the summer solstice to when recrudescence commenced, 3 to 4 weeks after the winter solstice.

In contrast to the only other study of annual changes in testis volume of mature captive male P. scapulatus (McGuckin and 

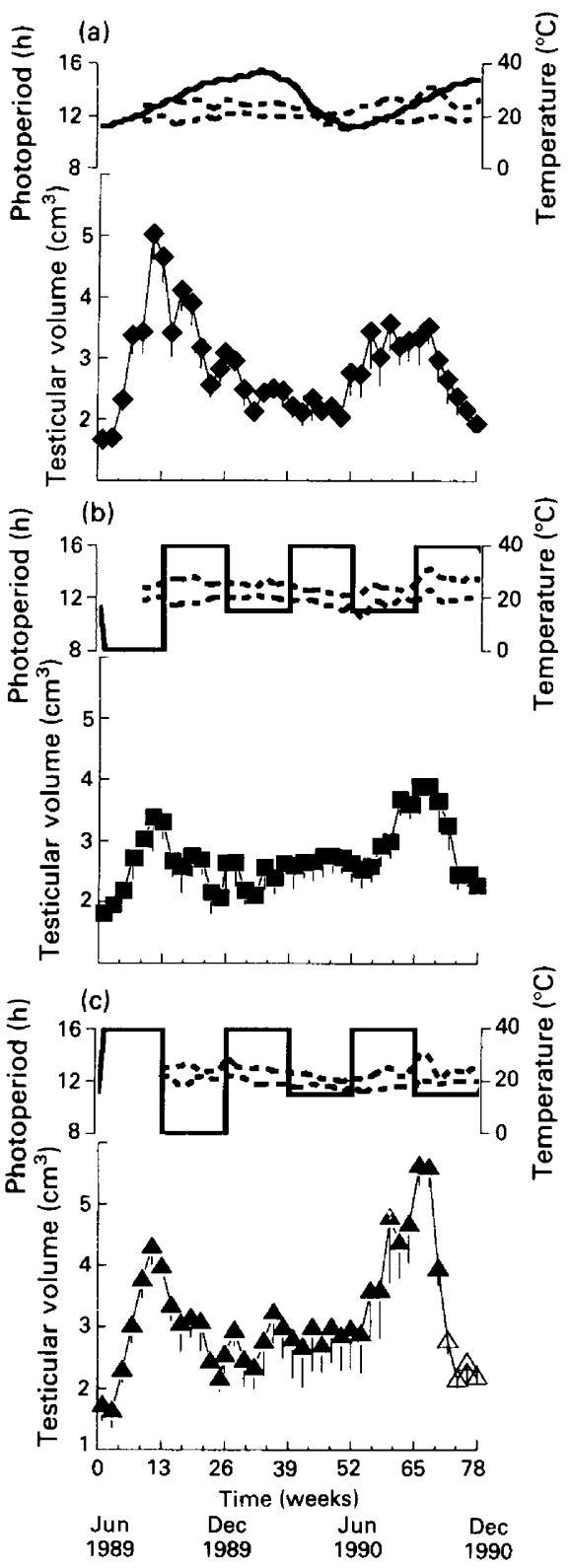

Fig. 3. Cycles of testis volumes in $P$. scapulatus maintained under (a) simulated natural photoperiod (Group A: $\bullet$ ); (b) and (c) alternating periods of short (1 $1 \mathrm{~h}$ light:13 $\mathrm{h}$ dark) or long (16 h light:8 $\mathrm{h}$ dark) daylength (Group B: $\mathbf{\square}$; Group C: $\boldsymbol{\Delta}$ ). Photoperiod treatments for Group $B$ and Group $C$ were three months out of phase. Data are means \pm $\mathrm{SEM},(\triangle)$ mean \pm range; $(\longrightarrow)$ photoperiod; (-- ) maximum and minimum temperatures.

Blackshaw, 1991), the present findings demonstrate a remarkably reproducible cycle, both in terms of amplitude and phase. The only data from wild populations for comparison are those from Nelson (1965), who monitored testis weights from December through April. Those data showed a slight downward trend, but as Fig. 2 shows regression would have been almost complete by the start of that period.

Body weight increased steadily during the year, with a sharp decline during testicular regression. The pattern was particularly well defined during the second year, June 1990-June 1991
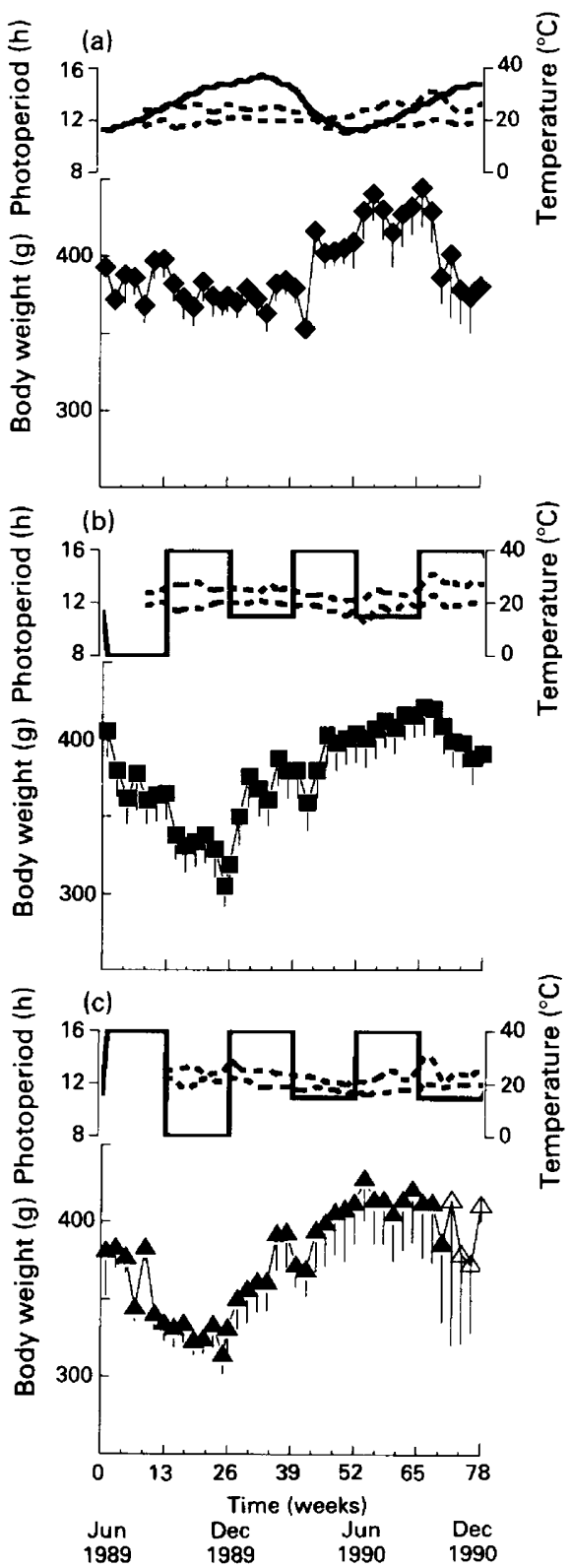

Fig. 4. Mean body weights of $P$. scapulatus maintained under (a) simulated natural photoperiod (Group A: $\bullet$ ); (b) and (c) alternating periods of short ( $11 \mathrm{~h}$ light:13 h dark) or long ( $16 \mathrm{~h}$ light:8 h dark) daylength (Group B: $\mathbf{\square}$; Group C: $\mathbf{\Delta}$ ). Photoperiod treatments for Group $B$ and Group $C$ were three months out of phase. Data are means \pm SEM, $(\triangle)$ mean \pm range; $(-)$ photoperiod; (-- ) maximum and minimum temperatures.

(Fig. 2b). McGuckin and Blackshaw (1991) also described a seasonal drop in body weight at the time of mating, both in $P$. scapulatus and $P$. poliocephalus.

Photoperiod manipulation did not disrupt the seasonal cycle of testicular growth in P. scapulatus. The test groups (Groups B and $C$ ) did not cycle out of phase, and neither group became entrained to the experimental photoperiod regimen, remaining in synchrony with the indoor controls (Group A) throughout. Abrasions of sebaceous neck glands that are used for 'marking' territories were noted during both periods of testicular growth, 
but were absent during the intervening months (G. M. O'Brien, unpublished). Thus changing photoperiod is not necessary for synchronization of the breeding season in the males of this species. Nor is it necessary for supporting full expression of the testicular cycle, as the maximum testis size achieved by Group $C$ in the second year matched that of the normal breeding males in natural photoperiod; and Group $\mathrm{A}$, which did receive changing photoperiod, did not achieve the same maximum testis size.

The body weight changes of Groups A and B indicate a trend toward weight increasing in time for breeding and weight loss during and after the breeding season, even in the absence of breeding opportunities. The pattern was less well defined in Group C. While the prolonged period of declining body weight (June to December, 1989) may have modified responses of Groups B and C during the first cycle of photoperiod, the presence of normal weights in the absence of response during the second cycle make this an unlikely explanation.

Species that are reproductively photoresponsive usually respond to a photoperiod change within two months, and in the ram alternating periods of short and long daylengths will entrain the cycle of testicular growth, as long as the duration of each cycle remains greater than 4 months (Pelletier and Almeida, 1987). Since $P$. scapulatus had shown no indication of entrainment to three photoperiod cycles of 6 months duration, it is reasonable to conclude that they are not reproductively photoresponsive. However, if a single light step each three months was insufficient drive to the reproductive axis/ hypothalamic clock in the present study, a 'normal' sine wave pattern with incremental changes in photoperiod may have a different effect. The possibility remains, therefore, that for this species, the photoperiod treatment used here was too different from a normal year for the hypothalamus to be able to respond. That the experimental design used here, of alternating 3 month periods of short followed by long days, in constant temperature and segregated groups, can induce changes in a photoresponsive species, has been confirmed in another species of flying fox. $P$. poliocephalus males were gradually entrained by the applied photoperiod such that, in the second year, they expressed two cycles of testicular growth (O'Brien et al., 1991). McGuckin and Blackshaw (1992) were also able to modify the reproductive cycle of $P$, poliocephalus, using a pulse of long days, lasting 2 to 4 months, followed by short days.

Most sources of environmental information other than photoperiod can also be largely eliminated as possible cues entraining the annual reproductive rhythm in male $P$. scapulatus. In the present experiment, Groups A, B and C were housed indoors for photoperiod manipulation. Thereby, they were removed from the influences of females, fluctuations in temperature, precipitation, moonlight, and all parameters of sunlight (possible seasonal changes in wavelengths, intensities, northsouth axis, quality and duration of twilight), but they maintained the same time course of testicular cycles as the outdoor breeding group. All groups received identical fruit diets, but it is unlikely that this provided synchronizing cues: potential information about seasons have been largely masked by market forces that have led to many fruits being available continuously rather than seasonally. While it would be desirable to hold diet constant throughout the experiment, the need for large quantities of fresh fruit over several years makes this impracticable. Instead, food sources used by free-living populations - blossom and native fruits - were entirely excluded.

$P$. scapulatus are nomadic, located mainly in the tropics during winter and early spring, but widely dispersed from October to May (Nelson, 1965; McWilliam, 1985). The time course of the testicular cycle defined here shows that recrudescence would be initiated and almost complete while animals are in tropical regions where daylength changes are relatively small.

Many tropical species are seasonal breeders, but none has been shown to use photoperiod to synchronize their natural reproductive cycles. Seasonal breeders (Heideman and Bronson, 1990a), continuous breeders (Heideman and Bronson, 1990b) and opportunistic breeders (Rissman et al., 1987) from tropical regions can monitor daylength with melatonin secretion, but their neuroendocrine pathways do not use this information to regulate breeding seasons. From the current investigation it appears that $P$. scapulatus resemble seasonally breeding tropical mammals, although their distribution extends well into temperate zones (at least for part of the year). The seasonal cycle of testicular growth and regression of male $P$. scapulatus is not immediately regulated by photoperiod, although the need remains to test them with less dramatic shifts in photoperiod.

If the present finding of the absence of a photoperiodic response of the reproductive cycle of $P$. scapulatus is confirmed in tests using alternative protocols, then $P$. scapulatus may be the first species found that maintains a 12 month testicular cycle in the presence of a photoperiodic challenge. This leads to the important consideration that their reproduction may be regulated by a circannual rhythm. The presence of a circannual rhythm would imply that there had been a period when the hypothalamic clock was more plastic than it is in adulthood, and may have been influenced by, for example, the duration of gestation, time of birth, photoperiodic experience at puberty, or social entrainment since birth. These parameters remain to be investigated.

This work was supported by an Australian Research Council grant to L. Martin and A. W. Blackshaw, and a National Research Fellowship to G. M. O'Brien. The assistance of L. M. Little and P. A. Clarke (animal maintenance) and the inspectors of the Department of Primary Industries (animal feed) are gratefully acknowledged.

\section{References}

Bhatnagar KP, Frahm HD and Stephan H (1990) The megachiropteran pineal organ: a comparative morphological and volumetric investigation with special emphasis on the remarkably large pineal of Dobsonia praedatrix Journal of Anatomy 168 143-166

Hall LS (1987) Identification, distribution and taxonomy of Australian flying-foxes (Chiroptera: Pteropodidae) Australian Mammalogy 10 75-79

Heideman PD and Bronson FH (1990a) Reproductive responses to photoperiod in tropical mammals Biology of Reproduction (Supplement 1) 42153

Heideman PD and Bronson FH (1990b) Photoperiod, melatonin secretion, and sexual maturation in a tropical rodent Biology of Reproduction 43 745-750

McGuckin MA (1988) Seasonal changes in the reproductive physiology of male flying foxes (Pteropus spp.) in South East Queensland PhD Thesis, University of Queensland

McGuckin MA and Blackshaw AW (1988) Different responses to the same environmental cues regulate long and short day breeding seasons in two species of Pteropus (Megachiroptera) in South-East Queensland Proceedings of the International Congress on Animal Reproduction and Artificial Insemination 11 412 
McGuckin MA and Blackshaw AW (1991) Seasonal changes in testicular size, plasma testosterone concentration and body weight in captive flying foxes (Pteropus poliocephalus and P. scapulatus) Journal of Reproduction and Fertility 92 339-346

McGuckin MA and Blackshaw AW (1992) The effects of photoperiod on the reproductive physiology of male flying foxes, Pteropus poliocephalus Reproduction, Fertility and Development 4 43-53

McWilliam AN (1985) The feeding ecology of Pteropus in north-eastern New South Wales, Australia Myotis 23-24 201-208

Nelson JE (1965) Movements of Australian flying foxes (Pteropodidae: Megachiroptera) Australian Journal of Zoology 13 35-73

O'Brien GM, Curlewis JD and Martin L (1991) Unusual reproductive photoresponsiveness of male greyheaded flying foxes (Pteropus poliocephalus) Proceedings of the Australian Society for Reproductive Biology 23160
Pelletier J and Almeida G (1987) Short light cycles induce persistent reproductive activity in lle-de-France rams Joumal of Reproduction and Fertility Supplement 34 215-226

Ratcliffe FN (1931) Notes on the fruit bats (Pteropus spp.) of Australia Journal of Animal Ecology 1 32-57

Rissman EF, Nelson RJ, Blank JL and Bronson FH (1987) Reproductive response of a tropical mammal, the musk shrew (Suncus murinus), to photoperiod Journal of Reproduction and Fertility 81 563-566

SAS (1985) SAS Proprietary Software Release 6.04 SAS Institute, Cary, NC

Selye $\mathbf{H}$ (1953) The diseases of adaptation: introductory remarks Recent Progress in Hormone Research 8117 